\title{
Influence of serotonin receptor 2A His452Tyr polymorphism on brain temporal structures: a volumetric MR study
}

\author{
Nicola Filippini ${ }^{1}$, Catia Scassellati ${ }^{2}$, Marina Boccardi ${ }^{1}$, Michela Pievani ${ }^{1}$, Cristina Testa ${ }^{1,3}$, \\ Luisella Bocchio-Chiavetto ${ }^{2}$, Giovanni B Frisoni*,1,4,5 and Massimo Gennarelli ${ }^{2,6}$
}

\begin{abstract}
${ }^{1}$ LENITEM Laboratory of Epidemiology, Neuroimaging, \& Telemedicine, Brescia, Italy; ${ }^{2}$ Genetic Unit, Brescia, Italy; ${ }^{3}$ Machine Vision Laboratory, Department of Mathematics and Computer Science, University of Udine, Udine, Italy; ${ }^{4}$ Psychogeriatric Ward I.R.C.C.S. 'San Giovanni di Dio', Fatebenefratelli, Brescia, Italy; ${ }^{5}$ AFaR Associazione Fatebenefratelli per la Ricerca, Rome, Italy; ${ }^{6}$ Division of Biology and Genetics, Department of Biomedical Sciences and Biotechnologies, University of Brescia, Brescia, Italy
\end{abstract}

Serotonin (5-HT) receptors $2 \mathrm{~A}$ are expressed in brain regions involved in memory and learning processes. Recently, a functional single nucleotide polymorphism in the 5-HT2A receptor gene leading to an aminoacid substitution at residue 452 (His452Tyr) has been involved in memory performance, persons with the rare 452Tyr allele showing poorer memory performance compared to His452His subjects. To investigate a putative structural effect of this polymorphism on temporal areas typically involved in memory processes, we performed voxel-based morphometry (VBM) and region-of-interest (ROI) volumetric analysis on highresolution magnetic resonance images in 15 carriers and 61 noncarriers of the 452Tyr allele. ROI volumetric analysis showed a significant reduction of the fractional volume of the temporal white matter in 452Tyr carriers $(0.67 \pm 0.07$ vs $0.73 \pm 0.08 ; P=0.007)$. VBM confirmed this finding and in addition showed reduced grey matter in the left hippocampus, left inferior temporal gyrus, and bilaterally in the middle and superior temporal gyrus. A possible effect on synaptic plasticity or neurodevelopment might explain the influence of the His452Tyr polymorphism on temporal brain structures, and this might be the basis for poorer memory performance in $452 \mathrm{Tyr}$ carriers. These findings should be considered preliminary and future replication is needed.

European Journal of Human Genetics (2006) 14, 443-449. doi:10.1038/sj.ejhg.5201573; published online 25 January 2006

Keywords: Hys452Tyr; 5-HT2A receptor; MR; temporal structures; neuroimaging

\section{Introduction}

Serotonin (5-hydroxytryptamine, 5-HT) and its receptors are widely distributed in the central nervous system (CNS) ${ }^{1}$ and influence several behaviours such as food intake, sexual behaviour, activity rhythms, and emotional states, ${ }^{2}$

${ }^{*}$ Correspondence: Dr GB Frisoni, LENITEM Laboratory of Epidemiology, Neuroimaging, \& Telemedicine, IRCCS S. Giovanni di Dio - FBF, Via Pilastroni, 4-25125 Brescia, Italy. Tel: + 39030350 1361;

Fax: + 39030353 3513; E-mail: papers@centroAlzheimer.it

Received 1 July 2005; revised 7 November 2005; accepted 7 December 2005; published online 25 January 2006 as well as higher cognitive functions such as learning and memory processes. ${ }^{3,4}$ In particular, 5-HT2A receptors are located mainly in the prefrontal cortex and hippocampus, ${ }^{5,6}$ which are key brain structures for memory. ${ }^{7}$

To date, associations of genetic polymorphisms with brain morphology have been widely reported. ${ }^{8-12}$ Recently, a functional single nucleotide polymorphism (SNP) in the 5-HT2A receptor gene leading to an aminoacid substitution at residue 452 (His452Tyr) has been found. The common variant is the $\mathrm{C}$ allele (His452His) and the rare is the 452Tyr allele (His452Tyr), with a 
frequency of $9.3 \%$ in Caucasian populations. ${ }^{13}$ Recent findings have demonstrated a functional influence of this SNP on human memory, 452Tyr carriers showing $21 \%$ poorer memory performance than noncarriers. ${ }^{14}$ In this study, healthy young volunteers viewed six sets of semantically unrelated words and performed an incidental episodic memory task with immediate and delayed recall after $5 \mathrm{~min}$ and $24 \mathrm{~h}$. In both conditions, 452Tyr carriers showed lower memory performance compared to noncarriers.

Moreover, it has been shown that this effect is modified by age in that it can be detected in young to middle-aged, but not in older persons. ${ }^{15}$

Aim of this study was to investigate whether the 452Tyr allele is associated with morphological changes in the normal brain, particularly in the temporal structures typically involved in memory processes, such as the hippocampus and parahippocampal grey and white matter (GM and WM). We addressed this question by studying high-resolution structural magnetic resonance (MR) images with region-of-interest (ROI) and voxel-based morphometry (VBM) volumetric analyses in carriers and noncarriers of the allele. VBM is a recently developed technique allowing to study the effect of different factors on brain structure with exquisite detail. ${ }^{16}$ Whole brain maps can be drawn that indicate where atrophic changes occur with a precision of a few millimetres.

\section{Materials and methods Subjects}

A total of 76 healthy subjects (28 males and 48 females) aged 40 years and older, living in Northern Italy, were enrolled in a study on normal brain structure with MR as described in detail elsewhere. ${ }^{17}$ Briefly, subjects were picked among those undergoing brain MR scan at the Neuroradiology Unit of the 'Città di Brescia' Hospital from March 2001 to March 2003 for reasons other than memory disturbance, cognitive impairment, degenerative diseases, or head trauma and whose MR scan was negative. The reasons for MR prescription were generally migraine and headache, auditory (hypoacusia, dizziness, tinnitus) or visual concerns (diplopia), sensory disturbances (paresthesias), suspected cerebrovascular disease, and miscellaneous (dyslexia, orbit study, lipotimic episodes, etc). Exclusion criteria were based on information before MR findings. Clinical exclusion criteria were: (1) MR scan for memory problems or cognitive impairment, (2) MR scan for clinical suspicion of neuro-degenerative diseases (Parkinson's disease, progressive supranuclear palsy, Huntington's disease, multiple system atrophy, etc), (3) patient undergoing MR for suspected stroke, (4) history of TIA or stroke, head trauma, alcohol and substance abuse, cortico-steroid therapy, and loss of weight greater than $5 \mathrm{~kg}$ in the last 6 months, and (5) cognitive impairment on neuropsychological testing. Radiological exclusion criteria included: (1) brain mass, (2) WM hyperintensities in a subject undergoing MR for suspected multiple sclerosis, (3) aneurysm larger than $10 \mathrm{~mm}$, (4) arteriovenous malformation (except for small developmental venous anomalies), and (5) malformations of the CNS.

Subjects were intercepted in the waiting room of the neuroradiology unit, were explained aim and methods, and were asked to take part in the study. All subjects underwent multidimensional assessment including clinical, neurological and neuropsychological evaluations, and drawing of a blood sample. The study was approved by the local ethics committee.

\section{5-HT2A His452Tyr polymorphism}

Genomic DNA was isolated from peripheral white blood cell samples by Puregene kit (Gentra System), according to the manufacturer's protocol.

The 5-HT2A His452Tyr polymorphism changes a cleavage site for a specific restriction endonuclease and, after PCR amplification, was analysed through restriction enzyme BseXI (Fermentas) as described by Erdmann et al. ${ }^{18}$

\section{MR imaging}

Three-dimensional (3D) high-resolution T1-weighted MR images were acquired on a 1.0 Tesla Philips Gyroscan $\left(\mathrm{TR}=20 \mathrm{~ms}, \quad \mathrm{TE}=5.0 \mathrm{~ms}, \quad\right.$ flip angle $=30^{\circ}$, field of view $=220 \mathrm{~mm}$, acquisition matrix $256 \times 256$, slice thickness $1.3 \mathrm{~mm}$ ).

MR images were processed with SPM99 (http://www.fil. ion.ucl.ac.uk/spm) following an optimized protocol including: (a) generation of a whole brain customized template, (b) generation of customized prior probability maps, and (c) main VBM steps based on the previous customized images.

Customized template This was obtained by normalizing MR images to the Montreal Neurological Institute (MNI) template $^{19}$ of SPM99 using a 12-parameter affine transformation, smoothing the normalized images with an $8 \mathrm{~mm}$ isotropic Gaussian kernel and averaging the smoothed images. The anterior commissure (AC) was manually set as the origin of the spatial coordinates for the normalization algorithm: ${ }^{20}$ AC coordinates were detected through the 'Display' window by an investigator blinded to gene status, and were used to modify the origin using the 'HDR edit' button. The normalization uses a bilinear interpolation algorithm to reslice images to voxel size of $2 \times 2 \times 2 \mathrm{~mm}^{3}$. This voxel size will be used in the following processing and analysis. All steps were visually checked with the 'Check reg' routine.

Customized prior probability maps These were computed by segmenting the normalized images into GM, WM, and cerebrospinal fluid (CSF), then smoothing with 
an $8 \mathrm{~mm}$ Gaussian filter, and finally averaging the segmented images, thus obtaining the customized prior probability maps specific for GM, WM, and $\mathrm{CSF}^{21}$

Main VBM steps Original MR images were normalized to the whole brain customized template through affine and nonlinear transformations with $7 \times 8 \times 7$ basis functions, medium regularization, and reslicing $2 \times 2 \times 2 \mathrm{~mm}^{3}$. The normalized images were segmented into GM, WM, and CSF using the customized prior probability maps. The ' $\mathrm{X}$-brain' routine, based on erosions and dilatations, was used to remove voxels of non-brain tissue from the segmented images, thus obtaining a brain image for each subject. This was used to clean GM, WM, and CSF images. In the modulation step, voxel values of the cleaned GM images were multiplied by the measure of relative volumes of warped and unwarped structures derived from the nonlinear step of spatial normalization (Jacobian determinant). ${ }^{22}$ The modulated images were smoothed with an $12 \mathrm{~mm}$ isotropic Gaussian kernel.

\section{ROI analysis}

In order to compute global and lobar volumes, a customized program (http://www.jiscmail.ac.uk/cgi-bin/wa.exe? $\mathrm{A} 2=\mathrm{ind} 02 \& \mathrm{~L}=\mathrm{spm} \& \mathrm{P}=\mathrm{R} 176348 \& \mathrm{I}=-1$ ) was applied to GM, WM, and CSF modulated images. Modulated images are $3 \mathrm{D}$ matrices where the intensity of each voxel is proportional to GM, WM, and CSF volume within each voxel. The program calculates volumes by summing the voxels of the modulated images times voxel volumes. The total intracranial volume (TIV) was computed as the sum of GM, WM, and CSF volumes.

ROI volumes were computed by applying a binary mask to the modulated images. The mask was traced with MRIcro along the boundaries of the temporal lobe on the mean of normalized MR images, ${ }^{23,24}$ and volumes were calculated as described above and normalized for TIV.

\section{VBM analysis}

SPM99 was used to carry out an ANCOVA, to detect atrophic regions of 452 Tyr carriers compared to noncarriers. Proportional scaling was set at 0.8 , and age, sex, and intracranial volume were included as nuisance covariates. A small volume correction procedure was used through a binary lobar mask outlining the temporal lobes. $P$ was set at 0.05 with small volume correction. An uncorrected confirmatory full brain analysis was performed with significance threshold at $P=0.001$, in order to check that significant clusters were confined to the temporal ROIs.

\section{Statistics}

Hardy-Weinberg equilibrium was tested by the Arlequin software version $2.000 .^{25}$

Despite the low number of His452Tyr carriers, parametric tests ( $T$ test) were used for brain measurement as values were normally distributed, as shown with Shapiro-Wilk test.

\section{Results}

Genotype frequencies were in Hardy-Weinberg equilibrium $(P=0.59)$. The frequency of the 452 Tyr allele was 0.105 , similar to the value reported for Caucasian populations $^{13}\left(\chi^{2}=0.125\right.$, d.f. $\left.=2, P=0.72\right)$, genotypic frequencies of His452His, His452Tyr, and Tyr452Tyr being 0.803 (61/ 76), 0.184 (14/76), and $0.013(1 / 76)$, respectively.

452Tyr carriers and noncarriers had similar age, sex, and education (Table 1). The clinical reasons leading to the prescription of the MR exam - migraine and headache (28\%), auditory (38\%) or visual (7\%) symptoms, paresthesia $(5 \%)$, suspected cerebrovascular disease $(6 \%)$, and miscellaneous reasons $(16 \%)$ - were homogeneously distributed between carriers and noncarriers $\left(\chi^{2}=5.455\right.$, d.f. $=5, P=0.409$ ).

The normality of the brain variables has been assessed with Shapiro-Wilk test (left temporal GM: d.f. $=15$,

Table 1 Socio-demographics and clinical features of carriers and noncarriers of the His452Tyr polymorphism are described in detail

\begin{tabular}{lccc}
\hline & His 452 Tyr carriers & Noncarriers & $P$ \\
\hline Age (years) & $53.80(9.48)$ & $54.86(11.07)$ & 0.87 \\
Education (years) & $10.47(2.85)$ & $10.13(4.43)$ & 0.69 \\
Sex, (women) & $12(75 \%)$ & $36(60 \%)$ & 0.15 \\
MMSE & $28.90(1.19)$ & $29.33(.899)$ & 0.16 \\
Hypercholesterolemia & $11(73 \%)$ & $36(59 \%)$ & 0.38 \\
Diabetes & 0 & $3(4.9 \%)$ & 1 \\
Hypertension & $4(26 \%)$ & $15(24 \%)$ & 1 \\
Family history for dementia & $5(33 \%)$ & $14(23 \%)$ & 0.50 \\
Handedness & 0 & $3(4.9 \%)$ & 1 \\
\hline
\end{tabular}

Values denote mean (standard deviation) or numbers of subjects (percentage); $P$-values were performed with Mann-Whitney test for continuous and with Fisher's test for the dichotomous variables; MMSE: Mini Mental State Examination; Handedness: based on Edinburgh Handedness Inventory. 
$P=0.226$; right temporal GM: d.f. $=15, P=0.529$; left temporal WM: d.f. $=15, P=0.397$; right temporal GM: d.f. $=15, P=0.971)$.

ROI volume analysis indicated a significant reduction of the WM in the left temporal lobe. It should be highlighted that, although not reaching the threshold for statistical significance, the point estimates of lobar volumes of the right WM and bilateral GM indicated smaller volumes in carriers compared to noncarriers (Table 2).

VBM analysis showed that 452Tyr carriers had reduced GM volume in the left hippocampus and left inferior temporal gyrus and, bilaterally, in the middle and superior temporal gyrus (Figure 1 and Table 3). The WM was reduced in the left temporal regions (superior temporal

Table 2 Region of interest (ROI) volumetric analysis: temporal GM and WM volume in His452Tyr polymorphism carriers compared to noncarriers

\begin{tabular}{llll}
\hline & His452Tyr carriers & Noncarriers & $P$ \\
\hline Grey matter & & & \\
R & $3.16(0.27)$ & $3.22(0.21)$ & 0.32 \\
L & $3.03(0.21)$ & $3.11(0.24)$ & 0.25 \\
White matter & & & \\
R & $0.73(0.08)$ & $0.77(0.08)$ & 0.09 \\
L & $0.67(0.07)$ & $0.73(0.08)$ & 0.007 \\
\hline
\end{tabular}

$\mathrm{L}=$ left, $\mathrm{R}=$ right.

Numbers denote mean (standard deviation) of lobar volumes corrected by total intracranial volume (TIV); $P$-values were performed with $T$-test.
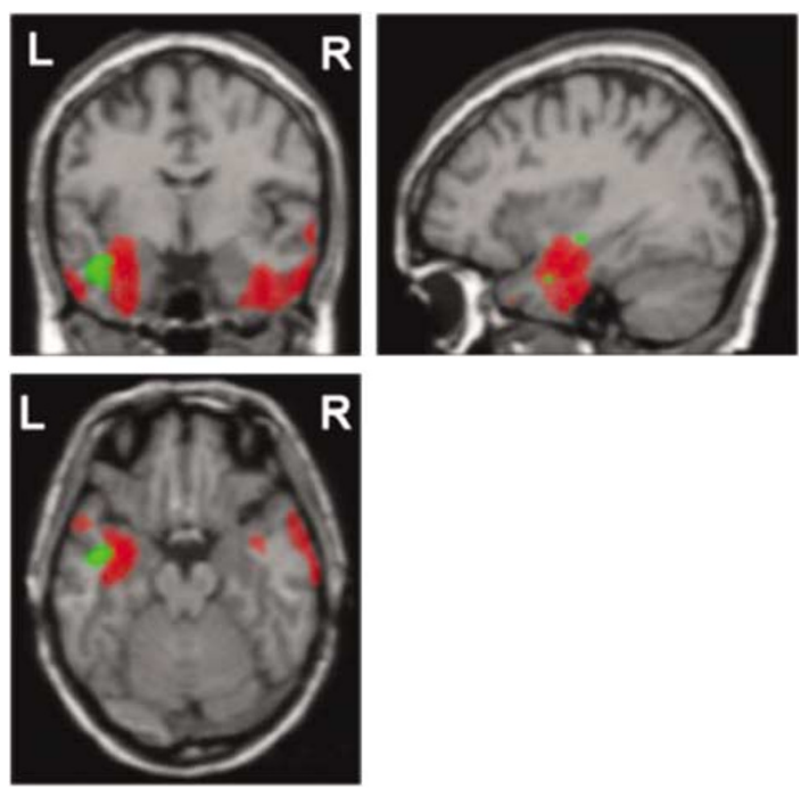

Figure $1 \mathrm{VBM}$ analysis at $P=0.05$ corrected with small volume correction showing volume reduction in carriers $v s$ noncarriers of the 452Tyr allele. $\mathrm{L}=$ left; $\mathrm{R}=$ right. Red spots indicate $\mathrm{GM}$ and green spots indicate WM regions of atrophy in subjects carrying the 452Tyr allele. sulcus and gyrus) in the 452Tyr carriers, contiguous to the clusters of GM reduction.

Confirmatory whole-brain VBM analysis showed that volume differences were confined exclusively to the temporal regions (Figure 2). The opposite comparison did not detect any clusters of atrophy in the regions of interest.

\section{Discussion}

This is the first study investigating the relationship between 5-HT2A receptor polymorphism and brain structure. The VBM findings show an effect of the 452Tyr allele on temporal brain structures, carriers showing a reduction of the GM volume in the left hippocampus and inferior temporal gyrus (Brodmann area 20), and in the middle and superior temporal gyri bilaterally (Brodmann areas 21 and 22). A significant reduction of the WM is also evident in the left temporal lobe, in a region adjacent to the hippocampal formation. The ROI findings show a significant reduction of the $\mathrm{WM}$ in the left temporal lobe and smaller volumes of the right WM and bilateral GM in carriers compared to noncarriers of the His452Tyr polymorphism. These findings are obtained with two methods that provide complementary information about a possible loss or reduction of volume, ROI analysis showing a widespread and VBM showing more localized changes. Indeed, the results show widespread reduction of the WM in the left temporal lobe, detected by ROI, and a more localized reduction of the GM, detected by VBM analysis.

These findings are consistent with the poorer cognitive performance of 452Tyr carriers showed by recent studies. ${ }^{14,15}$ Such reduction of performance, observed for memory function, has actually been described for subjects younger than those in our sample. Our neuropsychological battery was not conceived to investigate in great detail memory function; therefore, a possible difference in performance might have not been detected in our study. Alternatively, older subjects might not display such difference at all. If this is the case, the smaller volume of brain tissue in the medial temporal regions might be associated to a lower potential capability for memory performance that might be compensated by alternative cognitive strategies in older people.

An effect on synaptic plasticity might explain the influence of the 452Tyr allele on temporal lobe structures, as suggested by studies showing a relationship of the 5-HT2A receptor with physiological processes closely related to synaptic plasticity such as, phospholipase C and $\mathrm{G}$ proteins activation, ${ }^{26}$ intracellular $\mathrm{Ca}^{2+}$ mobilization and release. ${ }^{27}$

The synaptic plasticity hypothesis is further supported by data indicating a role of 5-HT2A receptors in adult neurogenesis and synaptogenesis. Adult mammals cells proliferate in subgranular layer and tend to migrate to the granule cell layer of the dentate gyrus. ${ }^{28,29}$ These 
Table 3 VBM analysis: atrophic regions in carriers versus noncarriers of the 452Tyr allele of the 5HT-2A receptor gene $(P=0.05$ with small volume correction)

\begin{tabular}{|c|c|c|c|c|c|}
\hline \multirow[b]{2}{*}{ Cluster size } & \multirow[b]{2}{*}{ Region } & \multicolumn{4}{|c|}{ Stereotactic coordinates $(\mathrm{mm})$} \\
\hline & & $x$ & $y$ & $z$ & Z-score \\
\hline $\begin{array}{l}\text { Grey matter } \\
2867 \\
2186\end{array}$ & $\begin{array}{l}\text { L hippocampus } \\
\text { L inferior temporal gyrus } \\
\text { L middle temporal gyrus } \\
\text { L superior temporal gyrus } \\
\text { R middle temporal gyrus } \\
\text { R superior temporal gyrus }\end{array}$ & $\begin{array}{r}-34 \\
-60 \\
-54 \\
-58 \\
58 \\
62\end{array}$ & $\begin{array}{r}-4 \\
-10 \\
10 \\
10 \\
12 \\
8\end{array}$ & $\begin{array}{r}-22 \\
-34 \\
-34 \\
-8 \\
-32 \\
-2\end{array}$ & $\begin{array}{l}3.07 \\
3.04 \\
2.81 \\
2.70 \\
3.90 \\
2.15\end{array}$ \\
\hline $\begin{array}{l}\text { White matter } \\
720\end{array}$ & $\begin{array}{l}\mathrm{L} \text { superior temporal sulcus } \\
\mathrm{L} \text { superior temporal gyrus }\end{array}$ & $\begin{array}{l}-46 \\
-46\end{array}$ & $\begin{array}{r}-8 \\
-16\end{array}$ & $\begin{array}{r}-16 \\
-6\end{array}$ & $\begin{array}{l}3.47 \\
2.52\end{array}$ \\
\hline
\end{tabular}

$\mathrm{L}=$ left, $\mathrm{R}=$ right. Cluster size is in number of voxels.

Reading example: the first line denotes the presence of a 3D cluster made of 2867 contiguous voxels of significantly decreased GM volume. The most significant voxel of the cluster has stereotactic coordinates of $-34,-4,-22$, and is located in the left hippocampus. Within the same cluster there are other peaks of significance.

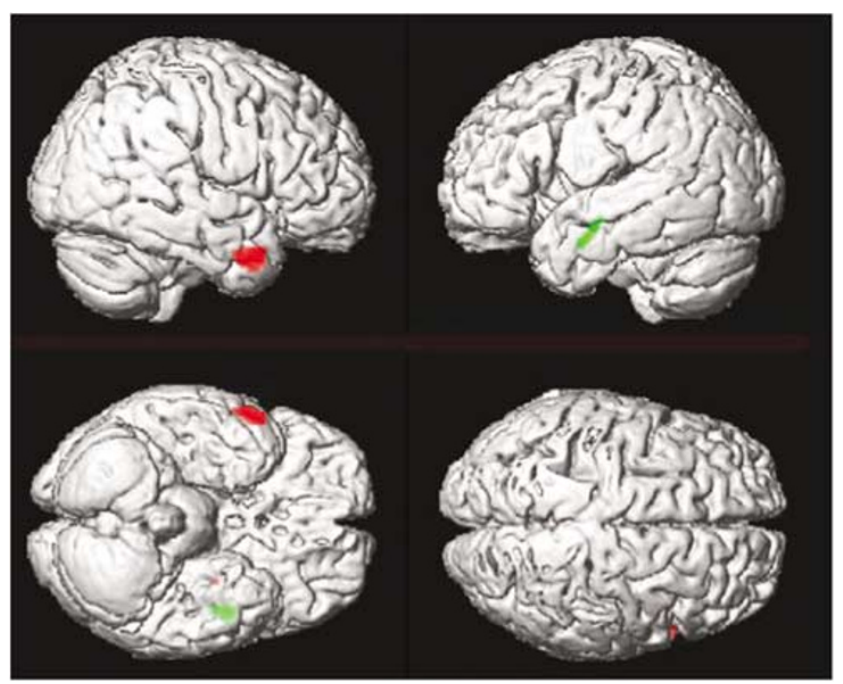

Figure 2 Whole-brain VBM analysis confirming that volume reductions of carriers $v$ s noncarriers of the $452 \mathrm{Tyr}$ allele were confined to the temporal regions. Maximal gray (red) and white (green) matter's $Z$-scores have been projected onto a brain surface $(P=0.001$ uncorrected).

'migrating' cells mature, grow dendrites, and establish synaptic contact within the hippocampus. ${ }^{30}$ Serotonin is one of the factors mostly involved in this process. ${ }^{31}$ Using systemic administration of 5-HT2A receptor antagonists in a mouse model of neurogenesis, a $63 \%$ reduction of the number of proliferating cells in the subgranular layer of the dentate gyrus has been shown, ${ }^{32}$ which consequently might determine a reduction in the cells maturation, in the growth of dendrites, and in the establishment of synaptic contact.

Data in the literature indicate that the 452Tyr allele might determine a reduced ability of 5-HT2A receptors to activate both phospholipase $\mathrm{C}$ and $\mathrm{G}$ proteins, ${ }^{26}$ which are known to have a role in long-term potentiation (LTP). ${ }^{33-35}$ LTP is regarded as one of the most popular and widely researched model involved in synaptic plasticity, ${ }^{36-40}$ which is also able to yield structural changes ${ }^{41}$ in hippocampal formation.

Moreover, it has been shown that 5-HT2A receptor development is associated with an increased $\mathrm{Ca}^{2+}$ availability in neurons, ${ }^{42}$ that in 452 Tyr carriers has been shown to be significantly reduced. ${ }^{24}$ This physiological process, acting on the internal cytoskeleton of the cell, is pivotal to cell proliferation and synaptogenesis, ${ }^{42}$ which are physiological processes interested in synaptic plasticity. ${ }^{43-46}$

Also, the 5-HT2A receptor has been shown to be involved in the regulation of BDNF mRNA in the hippocampus and neocortex. ${ }^{47}$ The administration of a 5-HT2A receptor agonist, the hallucinogenic phenylalkylamine 4-iodo-2, 5-dimethoxyphenylisopropylamine (DOI), showed a downregulation of BDNF mRNA levels in the frontal, parietal, and temporal cortex, particularly in the granule cell layer of the dentate gyrus. Indeed, it has been shown that BDNF mRNA expression in the hippocampal formation is influenced by stress, ${ }^{48}$ and animal studies have demonstrated a role of 5-HT2A receptors in mediating BDNF mRNA expression under stressful conditions ${ }^{49,50}$ supporting the involvement of 5-HT2A receptors in BDNF mRNA expression. It has been shown that a lower level of BDNF mRNA yields a decrease in neural plasticity. ${ }^{51,52}$ Subjects carrying the rare 452Tyr allele variant of the 5-HT2A receptor gene have a smaller peak amplitude in $\mathrm{Ca}^{2+}$ mobilization and release, ${ }^{27}$ which is related to a lower expression of BDNF $\mathrm{mRNA}^{53}$ in hippocampal neurons, and consequently it might lead to a decrease in neural plasticity.

Although the above-cited evidence converges in supporting the hypothesis that the different brain morphology 
observed in subjects with the 452Tyr allele might be due to an effect on neural plasticity, alternatives should not be disregarded. It might be hypothesized that persons with the 452Tyr allele have smaller brain volumes to start with. To date, very few studies have assessed the expression of 5-HT2A receptors in the embryo and their effect on neural development. One such a study has shown that 5-HT2A receptors affect morphogenesis of a variety of embryonic tissues such as the neuroepithelia of the brain and spinal cord, although specific studies on medial temporal structures are lacking. ${ }^{54}$ The neurodevelopmental hypothesis is particularly attractive to explain the effect of the 452Tyr allele on the WM where the absence of synapses makes the synaptic plasticity hypothesis less tenable.

Considering the small number of subjects in our sample, these findings should be considered preliminary and needing further confirmation with a larger number of subjects. Healthy volunteers selected randomly from the general population would be preferable than subjects intercepted in a neuroradiological department. The strict exclusion criteria we have employed should have allowed us to rule out conditions possibly related to both predictors (genotype) and outcome (regional volume changes). Indeed, although our experimental groups have a high prevalence of conditions, such as migraine and headache, these are not differentially distributed in carriers and noncarriers of the His452Tyr polymorphism and thus cannot be reported as confounders. The suggested hypotheses, of synaptic plasticity and neurodevelopment, might be better analysed studying the possible role of the His452Tyr polymorphism in the period of life between childhood and adulthood, in order to better understand its mechanism of action. These brain structural differences should also be confirmed with different brain measurement tools. The use of different scanners with a greater Tesla value (1.5/3.0) may improve the spatial resolution, in fact the higher is the Tesla value of the scanner, the better is the spatial, temporal, and spectral resolution and this may result in an improved accuracy. But it has also been noted a greater noise and also a higher susceptibility for artefacts, leading to geometric distortion and signal loss, particularly near the air-filled sinuses. ${ }^{55}$ Greater insight into the mechanisms through which genes influence brain structure and function certainly deserves deeper investigation.

\section{Acknowledgements}

This research was supported by grants from the Italian Ministry of Health (number: 00/343) and the CARIPLO Foundation.

\section{References}

1 Leysen JE: 5-HT2 receptors. Curr Drug Targets CNS Neurol Disord 2004; 3: 11-26.

2 Hoyer D, Clarke DE, Fozard JR et al: International union of pharmacology classification of receptors for 5-hydroxytryptamine (serotonin). Pharmacol Rev 1994; 46: 157-203.
3 Buhot MC: Serotonin receptors in cognitive behaviors. Curr Opin Neurobiol 1997; 7: 243-254.

4 Buhot MC, Martin S, Segu L: Role of serotonin in memory impairment. Ann Med 2000; 32: 210-221.

5 Meneses A: Involvement of $5-\mathrm{HT}(2 \mathrm{~A} / 2 \mathrm{~B} / 2 \mathrm{C})$ receptors on memory formation: simple agonism, antagonism, or inverse agonism? Cell Mol Neurobiol 2002; 22: 675-688.

6 Sheline YI, Mintun MA, Moerlein SM, Snyder AZ: Greater loss of 5-HT(2A) receptors in midlife than in late life. Am J Psychiatry 2002; 159: 430-435.

7 Squire LR, Zola-Morgan S: The medial temporal lobe memory system. Science 1991; 253: 1380-1386.

8 den Heijer T, Schuit SC, Pols HA et al: Variations in estrogen receptor alpha gene and risk of dementia, and brain volumes on MRI. Mol Psychiatry 2004; 9: 1129-1135.

9 Ho BC, Wassink TH, O'Leary DS, Sheffield VC, Andreasen NC: Catechol-O-methyl transferase Val158Met gene polymorphism in schizophrenia: working memory, frontal lobe MRI morphology and frontal cerebral blood flow. Mol Psychiatry 2005; 10: 287-298.

10 Pezawas L, Verchinski BA, Mattay VS et al: The brain-derived neurotrophic factor val66met polymorphism and variation in human cortical morphology. J Neurosci 2004; 24: 10099-10102.

11 Hariri AR, Mattay VS, Tessitore A et al: Serotonin transporter genetic variation and the response of the human amygdala. Science 2002; 297: 400-403.

12 Pezawas L, Meyer-Lindenberg A, Drabant EM et al: 5-HTTLPR polymorphism impacts human cingulate-amygdala interactions: a genetic susceptibility mechanism for depression. Nat Neurosci 2005; 8: 828-834.

13 Ozaki N, Rosenthal NE, Pesonen U et al: Two naturally occurring amino acid substitutions of the 5-HT2A receptor: similar prevalence in patients with seasonal affective disorder and controls. Biol Psychiatry 1996; 40: 1267-1272.

14 de Quervain DJ, Henke K, Aerni A et al: A functional genetic variation of the 5-HT2a receptor affects human memory. Nat Neurosci 2003; 6: 1141-1142.

15 Papassotiropoulos A, Henke K, Aerni A et al: Age-dependent effects of the 5-hydroxytryptamine-2a-receptor polymorphism (His452Tyr) on human memory. Neuroreport 2005; 16: 839-842.

16 Ashburner J, Csernansky JG, Davatzikos C, Fox NC, Frisoni GB, Thompson PM: Computer-assisted imaging to assess brain structure in healthy and diseased brains. Lancet Neurol 2003; 2: 79-88.

17 Riello R, Sabattoli F, Beltramello A et al: Brain volumes in healthy adults aged 40 years and over: a voxel based morphometry study. Aging Clin Exp Res 2005; 17: 329-336.

18 Erdmann J, Shimron-Abarbanell D, Rietschel M et al: Systematic screening for mutations in the human serotonin-2A (5-HT2A) receptor gene: identification of two naturally occurring receptor variants and association analysis in schizophrenia. Hum Genet 1996; 97: 614-619.

19 Evans AC, Kamber M, Collins DL, Macdonald D: An MRI-based probabilistic atlas of neuroanatomy; in Shorvon S, Fish D, Andermann F, Bydder GM, Stefan H (eds): Magnetic Resonance Scanning and Epilepsy. New York/London: Plenum press, 1994, pp 263-274.

20 Ashburner J, Neelin P, Collins DL, Evans AC, Friston KJ: Incorporating prior knowledge into image registration. Neuroimage 1997; 6: 344-352.

21 Good CD, Scahill RI, Fox NC et al: Automatic differentiation of anatomical patterns in the human brain: validation with studies of degenerative dementias. Neuroimage 2002; 17: 29-46.

22 Ashburner J, Friston KJ: Why voxel-based morphometry should be used. Neuroimage 2001; 14: 1238-1243.

23 DeCarli C, Maisog J, Murphy DG, Teichberg D, Rapoport SI, Horwitz B: Method for quantification of brain, ventricular, and subarachnoid CSF volumes from MR images. J Comput Assist Tomogr 1992; 16: 274-284.

24 DeCarli C, Murphy DG, Gillette JA et al: Lack of age-related differences in temporal lobe volume of very healthy adults. AJNR Am J Neuroradiol 1994; 15: 689-696. 
25 Schneider S, Roessli D, Excofier L: Arlequin: A Software for Population Genetics Data Analysis. Ver 2.000. Switzerland: Genetics and Biometry Laboratory Department of Anthropology, University of Geneva, 2000.

26 Hazelwood LA, Sanders-Bush E: His452Tyr polymorphism in the human 5-HT2A receptor destabilizes the signaling conformation. Mol Pharmacol 2004; 66: 1293-1300.

27 Ozaki N, Manji H, Lubierman V et al: A naturally occurring amino acid substitution of the human serotonin 5-HT2A receptor influences amplitude and timing of intracellular calcium mobilization. J Neurochem 1997; 68: 2186-2193.

28 Gage FH: Mammalian neural stem cells. Science 2000; 287: $1433-1438$.

29 Jacobs BL: Adult brain neurogenesis and depression. Brain Behav Immunol 2002; 16: 602-609.

30 Djavadian RL: Serotonin and neurogenesis in the hippocampal dentate gyrus of adult mammals. Acta Neurobiol Exp 2004; 64: $189-200$.

31 Brezun JM, Daszuta A: Depletion in serotonin decreases neurogenesis in the dentate gyrus and the subventricular zone of adult rats. Neuroscience 1999; 89: 999-1002.

32 Banasr M, Hery M, Printemps R, Daszuta A: Serotonin-induced increases in adult cell proliferation and neurogenesis are mediated through different and common 5-HT receptor subtypes in the dentate gyrus and the subventricular zone. Neuropsychopharmacology 2004; 29: 450-460.

33 Ballyk BA, Goh JW: A postsynaptic G-protein in hippocampal long-term potentiation. Brain Res 1993; 611: 81-86.

34 Satoh M, Kaneko S: Involvement of postsynaptic G-proteins in hippocampal long-term potentiation. Rev Neurosci 1994; 5: 1-9.

35 Cohen AS, Raymond CR, Abraham WC: Priming of long-term potentiation induced by activation of metabotropic glutamate receptors coupled to phospholipase C. Hippocampus 1998; 8: $160-170$

36 Bliss TV, Collingridge GL: A synaptic model of memory: longterm potentiation in the hippocampus. Nature 1993; 361: 31-39.

37 Larkman AU, Jack JJ: Synaptic plasticity: hippocampal LTP. Curr Opin Neurobiol 1995; 5: 324-334.

38 Holscher C: Synaptic plasticity and learning and memory: LTP and beyond. J Neurosci Res 1999; 58: 62-75.

39 Richter-Levin G, Yaniv D: Is LTP in the hippocampus a useful model for learning-related alterations in gene expression? Rev Neurosci 2001; 12: 289-296.

40 Muller D, Nikonenko I, Jourdain P, Alberi S: LTP, memory and structural plasticity. Curr Mol Med 2002; 2: 605-611.
41 Toni N, Buchs PA, Nikonenko I, Bron CR, Muller D: LTP promotes formation of multiple spine synapses between a single axon terminal and a dendrite. Nature 1999; 402: 421-425.

42 Azmitia EC: Modern views on an ancient chemical: serotonin effects on cell proliferation, maturation, and apoptosis. Brain Res Bull 2001; 56: 413-424.

43 Bourgeois JP: Brain synaptogenesis and epigenesis. Med Sci 2005 21: $428-433$

44 Munno DW, Syed NI: Synaptogenesis in the CNS: an odyssey from wiring together to firing together. J Physiol 2003; 552: $1-11$.

45 Cline HT: Dendritic arbor development and synaptogenesis. Curr Opin Neurobiol 2001; 11: 118-126.

46 Koo JW, Park CH, Choi SH: The postnatal environment can counteract prenatal effects on cognitive ability, cell proliferation, and synaptic protein expression. FASEB J 2003; 17: 1556-1558.

47 Vaidya VA, Marek GJ, Aghajanian GK, Duman RS: 5-HT2A receptor-mediated regulation of brain-derived neurotrophic factor mRNA in the hippocampus and the neocortex. $J$ Neurosci 1997; 17: 2785-2795.

48 Smith MA, Makino S, Kvetnansky R, Post RM: Stress and glucocorticoids affect the expression of brain-derived neurotrophic factor and neurotrophin-3 mRNAs in the hippocampus. J Neurosci 1995; 15: 1768-1777.

49 Vaidya VA, Terwilliger RM, Duman RS: Role of 5-HT2A receptors in the stress-induced down-regulation of brain-derived neurotrophic factor expression in rat hippocampus. Neurosci Lett 1999; 262: $1-4$.

50 Altar CA: Neurotrophins and depression. Trends Pharmacol Sci 1999; 20: 59-61.

51 Fossati P, Radtchenko A, Boyer P: Neuroplasticity: from MRI to depressive symptoms. Eur Neuropsychopharmacol 2004; 14: S503-S510.

52 Morimoto K, Sato K, Sato S, Yamada N, Hayabara T: Timedependent changes in neurotrophic factor mRNA expression after kindling and long-term potentiation in rats. Brain Res Bull 1998; 45: 599-605.

53 Tsuda M: Cascade of gene expression induced by Ca2+ signals in neurons. Neurochem Int 1996; 29: 443-451.

54 Lauder JM, Wilkie MB, Wu C, Singh S: Expression of 5-HT(2A), 5-HT(2B) and 5-HT(2C) receptors in the mouse embryo. Int J Dev Neurosci 2000; 18: 653-662.

55 Yang PC, Nguyen P, Shimakawa A et al: Spiral magnetic resonance coronary angiography - direct comparison of 1.5 Tesla vs 3 Tesla. J Cardiovasc Magn Reson 2004; 6: 877-884. 